\title{
Robust Control for Uncertain Switched Systems with Interval Nondifferentiable Time-Varying Delays
}

\author{
T. La-inchua ${ }^{1}$ and P. Niamsup ${ }^{1,2}$ \\ ${ }^{1}$ Department of Mathematics, Chiang Mai University, Chiang Mai 50200, Thailand \\ ${ }^{2}$ Center of Excellence in Mathematics, CHE, Si Ayutthaya Road, Bangkok 10400, Thailand \\ Correspondence should be addressed to P. Niamsup, piyapong.n@cmu.ac.th
}

Received 22 December 2011; Accepted 27 May 2012

Academic Editor: Vu Phat

Copyright (C) 2012 T. La-inchua and P. Niamsup. This is an open access article distributed under the Creative Commons Attribution License, which permits unrestricted use, distribution, and reproduction in any medium, provided the original work is properly cited.

This paper addresses the conditions for robust stabilization of a class of uncertain switched systems with delay. The system to be considered is autonomous and the state delay is time-varying. Using Lyapunov functional approach, restriction on the derivative of time-delay function is not required to design switching rule for the robust stabilization of switched systems with time-varying delays. The delay-dependent stability conditions are presented in terms of the solution of LMIs which can be solved by various available algorithms. A numerical example is given to illustrate the effectiveness of theoretical results.

\section{Introduction}

The switched system is a type of hybrid systems that consist of a family of a differential or difference equations and a switching rule to indicate which subsystem will be activated at a specific interval of time. For applications, switched systems can be used to describe several physical or chemical processes which are concerned by more than one dynamics: some systems work at some interval time then stop and other systems take over such as the automatic system in airplane, car energy system, traffic system, and machine industrial system, see [1,2]. Thus, a switching strategy must be designed in the study of stability of switched systems, also see [3-5].

The delay system has been considered in many research, especially the real processes in our world often involve time-delay; that is, the present state depends on the past states which brings more difficulty to investigate the stability of the system, especially time varying delay system, see [6-10]. In general, the following assumption on the derivative of the delay 
is made, namely $\dot{h}(t)<1$, see $[11,12]$ and references cited therein. This assumption may leads to conservativeness; for example, it might not be used when the delay is a fast or a nondifferential time varying function.

Moreover, in study of real world applications, the systems are in general influenced by disturbances which might cause inaccuracy of the data. The system can become unstable or less capable because of disturbance. Consequently, the study of robust stability of switched systems with time varying delay becomes important and has been studied by many researchers, see [7, 12-17].

In this paper, we study the problem of robust stability for a class of switched systems with time-varying delay. Compared with existing results in the literature, the novelty of our results is twofold. Firstly, the state delay is time-varying in which the restriction on the derivative of the time-delay function is not required to design switching rule in term of a dwell time for the robust stability of the system. Secondly, the obtained conditions for the robust stability are delay-dependent and formulated in terms of the solution of standard LMIs which can be solved by various available algorithms [18]. The paper is organized as follows. Section 2 presents notations, definitions, and auxiliary propositions required for the proof of the main results. Switching design for the robust stability of the system with illustrative examples is presented in Sections 3 and 4, respectively. The paper ends with a conclusion followed by cited references.

\section{Problem Formulation and Preliminaries}

Throughout this paper, the following notations will be used:

$\mathbb{R}^{n}$ - the $n$ dimensional Euclidean space;

$\mathbb{R}^{n \times n}$-the set of all $n \times n$ real matrices;

$\mathbb{N}$-the set of all positive integers;

$\|x\|$-the Euclidean norm of vector $x \in \mathbb{R}^{n}$;

$\operatorname{diag}\{\cdot\}$ —the block diagonal matrix;

$I$-the identity matrix;

$A^{T}$-the transpose of matrix $A$;

$A^{-1}$ - the inverse of matrix $A$;

$\left[\begin{array}{ll}A & B \\ * & C\end{array}\right]$-* the symmetric form of matrix, namely, $*=B^{T}$;

$\|A\|=\sup \left\{\|A x\|: x \in \mathbb{R}^{n},\|x\|=1\right\}$ for any $A \in \mathbb{R}^{n \times n} ;$

$C_{h_{2}}$-space of continuous vector-valued function defined on $\left[-h_{2}, 0\right]$;

$x_{t}(\theta)=x(t+\theta),-h_{2} \leq \theta \leq 0$, where $x_{t} \in C_{h_{2}} ;$

$\left\|x_{t}\right\|_{c_{h_{2}}}=\sup _{-h_{2} \leq \theta \leq 0}\|x(t+\theta)\| ;$

$\lambda_{\max }(A)=\max \{\operatorname{Re}(\lambda): \lambda$ is eigenvalue of $A\} ;$

$\lambda_{\min }(A)=\min \{\operatorname{Re}(\lambda): \lambda$ is eigenvalue of $A\} ;$

$M=\{1,2, \ldots, k\}$. 
Consider the following uncertain switched system with time varying delay:

$$
\begin{aligned}
\dot{x}(t)= & \left(A_{\sigma(t)}+\Delta A_{\sigma(t)}(t)\right) x(t)+\left(D_{\sigma(t)}+\Delta D_{\sigma(t)}(t)\right) x\left(t-h_{\sigma(t)}(t)\right) \\
& +\left(B_{\sigma(t)}+\Delta B_{\sigma(t)}(t)\right) u_{\sigma(t)}(t), \quad t>0, \\
x(t)= & \phi(t), \quad t \in\left[-h_{2}, 0\right],
\end{aligned}
$$

where $x(t)=\left[x_{1}(t), x_{2}(t), \ldots, x_{n}(t)\right]^{T} \in \mathbb{R}^{n}$ is the state vector. $A_{i}, D_{i}, B_{i}, i \in M$ are known constant matrices, $\Delta A_{i}, \Delta D_{i}, \Delta B_{i}$ are uncertainty matrices which are of the form

$$
\begin{array}{ll}
\Delta A_{i}=E_{1, i} F_{1, i}(t) H_{1, i}, & \Delta D_{i}=E_{2, i} F_{2, i}(t) H_{2, i}, \\
\Delta B_{i}=E_{3, i} F_{3, i}(t) H_{3, i}, & F_{j, i}^{T}(t) F_{j, i}(t) \leq I, \quad j=1,2,3,
\end{array}
$$

where $F_{1, i}(t), F_{2, i}(t), F_{3, i}(t)$ are unknown matrices, $I$ is the identity matrix of appropriate dimension. $h_{i}(t)$ is the delay function for an $i$ th subsystem which satisfies the following condition:

$$
0 \leq h_{1} \leq h_{1, i} \leq h_{i}(t) \leq h_{2, i} \leq h_{2}
$$

Let $\phi(t)$ be an initial condition. $\sigma(t): R^{+} \cup\{0\} \rightarrow M, t \in\left[t_{k}, t_{k+1}\right), k \in \mathbb{N}, \sigma(t)$ is called the switching signal; we have the switching sequence $\left\{x_{t_{0}} ;\left(i_{0}, t_{0}\right), \ldots \mid i_{k} \in M, k=0,1,2, \ldots\right\}$, which means that when $t \in\left[t_{k}, t_{k+1}\right)$, the $i_{k}$ th subsystem is activated.

Definition 2.1. $T^{*}=\inf \left\{t_{l}-t_{l-1}\right\}$ is called the dwell time of switched system.

Definition 2.2. The system (2.1) is said to be stabilizabled if there exists a feedback controller $u(t) \in \mathbb{R}^{m}$ such that the closed loop switched systems (without uncertainties) is asymptotically stable.

Definition 2.3. The system (2.1) is said to be robustly stabilizable if there exists a feedback controller $u(t) \in \mathbb{R}^{m}$ such that the closed loop uncertain switched systems are robustly stable.

The following lemmas will be used throughout this paper.

Lemma 2.4 (Schur complement lemma). Given constant symmetric matrices $Q, S$ and $R \in \mathbb{R}^{n \times n}$, where $R>0, Q=Q^{T}$, and $R=R^{T}$, one has

$$
\left[\begin{array}{cc}
Q & S \\
S^{T} & -R
\end{array}\right]<0 \Longleftrightarrow Q+S R^{-1} S^{T}<0
$$

Lemma 2.5 (see [13]). Given $\varepsilon>0$ and matrices $D, E$, and $F$ with $F^{T} F \leq I$, one has

$$
D E F+E^{T} F^{T} D^{T} \leq \varepsilon D D^{T}+\varepsilon^{-1} E^{T} E .
$$


Lemma 2.6 (see [13]). Given a positive definite matrix $P \in \mathbb{R}^{n \times n}$, any symmetric matrix $Q \in \mathbb{R}^{n \times n}$ and $x \in \mathbb{R}^{n}$, then

$$
\lambda_{\min }\left(P^{-1} Q\right) x^{T} P x \leq x^{T} Q x \leq \lambda_{\max }\left(P^{-1} Q\right) x^{T} P x .
$$

Lemma 2.7 (see [13]). For any positive semidefinite matrix $M \in \mathbb{R}^{n \times n}$, a scalar $\gamma>0$, and a vector function $\omega:[0, \gamma] \rightarrow \mathbb{R}^{n \times n}$ such that the integrals concerned are well-defined, one has

$$
\left(\int_{0}^{\gamma} \omega(s) d s\right)^{T} M\left(\int_{0}^{\gamma} \omega(s) d s\right) \leq \gamma \int_{0}^{\gamma} \omega^{T}(s) M \omega(s) d s
$$

Lemma 2.8 (Cauchy inequality). For any symmetric positive definite matrix $N \in M^{m \times m}$ and $x, y \in \mathbb{R}^{n}$, one has

$$
\pm 2 x^{T} y \leq x^{T} N x+y^{T} N^{-1} y
$$

For simplicity of later presentation, one gives the following notations.

$$
\begin{gathered}
\Sigma_{11, i}=P_{i} A_{i}^{T}+A_{i} P_{i}-B_{i} B_{i}^{T}+\left(2+h_{1}^{2}+h_{2}^{2}\right) Q_{i}-R_{i} ; \quad \Sigma_{12, i}=P_{i} A_{i}^{T}-0.5 B_{i} B_{i}^{T} ; \\
\Sigma_{13, i}=0.5 R_{i} ; \quad \Sigma_{14, i}=0.5 R_{i} ; \quad \Sigma_{15, i}=D_{i} P_{i} ; \quad \Sigma_{22, i}=\left(h_{1, i}^{2}+h_{2, i}^{2}\right) R_{i}+\delta_{i}^{2} Q_{i}-2 P_{i} ; \\
\Sigma_{25, i}=D_{i} P_{i} ; \quad \Sigma_{33, i}=-Q_{i}-0.5 R_{i}-0.5 U_{i} ; \quad \Sigma_{35, i}=0.5 U_{i} ; \quad \Sigma_{44, i}=-Q_{i}-0.5 R_{i}-0.5 U_{i} ; \\
\Sigma_{45, i}=0.5 U_{i} ; \quad \Sigma_{55, i}=-U_{i} ; \quad \lambda_{1}=\max _{i \in M}\left\{\lambda_{\max }\left(P_{i}^{-1}\right)\right\} ; \quad \lambda_{2}=\min _{i \in M}\left\{\lambda_{\min }\left(P_{i}^{-1}\right)\right\} ; \\
\lambda_{3}=\max _{i \in M}\left\{\lambda_{\max }\left(P_{i}\right)\right\} ; \quad \lambda_{4}=\min _{i \in I_{k}}\left\{\lambda_{\min }\left(-\Omega_{1, i}\right)\right\} ; \quad \lambda_{5}=\min _{i \in M}\left\{\lambda_{\min }\left(-\Omega_{2, i}\right)\right\} ; \\
\lambda^{*}=\min \left\{\lambda_{4}, \lambda_{5}\right\} ; \quad h_{a, i}=\frac{1}{2}\left(h_{1, i}+h_{2, i}\right) ; \quad \delta_{i}=\left(h_{2, i}-h_{1, i}\right) ; \quad h^{*}=\min _{i \in M}\left\{h_{2, i}-h_{a, i}\right\} ; \quad \delta=h_{2}-h_{1} .
\end{gathered}
$$

\section{Main Results}

\subsection{Asymptotical Stabilization for Nominal Switched Systems with Interval Time-Varying Delay}

The nominal switched systems are given by

$$
\begin{gathered}
\dot{x}(t)=A_{\sigma(t)} x(t)+D_{\sigma(t)} x\left(t-h_{\sigma(t)}(t)\right)+B_{\sigma(t)} u_{\sigma(t)}(t), \quad t>0 \\
x(t)=\phi(t), \quad t \in\left[-h_{2}, 0\right] .
\end{gathered}
$$

We now state the main result on sufficient condition for stabilization of the switched systems (3.1). 
Theorem 3.1. Given $\alpha \in(0,1)$. If there exists symmetric positive definite matrices $P_{i}, Q_{i}, R_{i}, U_{i}$ such that the following conditions hold:

$$
\begin{aligned}
& \Omega_{1, i}=\Omega_{i}-\left[\begin{array}{ll}
000 & -I I
\end{array}\right]^{T} U_{i}\left[\begin{array}{ll}
000 & -I I
\end{array}\right]<0, \\
& \Omega_{2, i}=\Omega_{i}-\left[\begin{array}{ll}
00 I 0 & -I
\end{array}\right]^{T} U_{i}\left[\begin{array}{ll}
00 I 0 & -I
\end{array}\right]<0, \\
& \Omega_{i}=\left[\begin{array}{ccccc}
\Sigma_{11, i} & \Sigma_{12, i} & \Sigma_{13, i} & \Sigma_{14, i} & \Sigma_{15, i} \\
* & \Sigma_{22, i} & 0 & 0 & \Sigma_{25, i} \\
* & * & \Sigma_{33, i} & 0 & \Sigma_{35, i} \\
* & * & * & \Sigma_{44, i} & \Sigma_{45, i} \\
* & * & * & * & \Sigma_{55, i}
\end{array}\right],
\end{aligned}
$$

and if

$$
T^{*} \geq \frac{1}{\rho} \ln \left(\frac{\lambda_{1}}{\alpha \lambda_{2}}\right)
$$

where $\rho=\min \left\{h^{*} \lambda^{*} / \delta \lambda_{3}, 1 / 2 h_{2}, 1 / 2\right\}, T^{*}$ is the dwell time, then for any switching rule satisfying (3.3) the switched system (3.1) is stabilizable under the feedback controller

$$
u_{i}(t)=-\frac{1}{2} B_{i}^{T} P_{i}^{-1} x(t), \quad t \geq 0
$$

Proof. Let $Y_{i}=P_{i}^{-1}, y(t)=Y_{i} x(t)$. Using the feedback controller (3.4), we choose a LyapunovKrasovskii functional candidates as

$$
V_{i}\left(x_{t}\right)=V_{1, i}\left(x_{t}\right)+\cdots+V_{8, i}\left(x_{t}\right)
$$

where

$$
\begin{aligned}
& V_{1, i}\left(x_{t}\right)=x^{T}(t) Y_{i} x(t), \\
& V_{2, i}\left(x_{t}\right)=\int_{t-h_{1, i}}^{t} x^{T}(s) Y_{i} Q_{i} Y_{i} x(s) d s, \\
& V_{3, i}\left(x_{t}\right)=\int_{t-h_{2, i}}^{t} x^{T}(s) Y_{i} Q_{i} Y_{i} x(s) d s, \\
& V_{4, i}\left(x_{t}\right)=h_{1, i} \int_{-h_{1, i}}^{0} \int_{t+s}^{t} \dot{x}^{T}(\theta) Y_{i} R_{i} Y_{i} \dot{x}(\theta) d \theta d s,
\end{aligned}
$$




$$
\begin{aligned}
& V_{5, i}\left(x_{t}\right)=h_{2, i} \int_{-h_{2, i}}^{0} \int_{t+s}^{t} \dot{x}^{T}(\theta) Y_{i} R_{i} Y_{i} \dot{x}(\theta) d \theta d s, \\
& V_{6, i}\left(x_{t}\right)=\delta_{i} \int_{-h_{2, i}}^{-h_{1, i}} \int_{t+s}^{t} \dot{x}^{T}(\theta) Y_{i} U_{i} Y_{i} \dot{x}(\theta) d \theta d s, \\
& V_{7, i}\left(x_{t}\right)=h_{1, i} \int_{-h_{1, i}}^{0} \int_{t+s}^{t} x^{T}(\theta) Y_{i} Q_{i} Y_{i} x(\theta) d \theta d s, \\
& V_{8, i}\left(x_{t}\right)=h_{2, i} \int_{-h_{2, i}}^{0} \int_{t+s}^{t} x^{T}(\theta) Y_{i} Q_{i} Y_{i} x(\theta) d \theta d s .
\end{aligned}
$$

It is easy to see that

$$
V_{i}\left(x_{t}\right) \geq c_{1}\|x(t)\|^{2},
$$

for some $c_{1}>0$. Taking the derivative of $V_{i}\left(x_{t}\right)$ with respect to $t$ along any trajectory of solution of (3.1) yields

$$
\begin{gathered}
\dot{V}_{1, i}\left(x_{t}\right)=2 x^{T}(t) Y_{i} \dot{x}(t), \\
=y^{T}(t)\left[P_{i} A_{i}^{T}+A_{i} P_{i}\right] y(t)-y^{T}(t) B_{i}^{T} B_{i} y(t)+2 y^{T}(t) D_{i} P_{i} y\left(t-h_{i}(t)\right), \\
\dot{V}_{2, i}\left(x_{t}\right)=y^{T}(t) Q_{i} y(t)-y^{T}\left(t-h_{1, i}\right) Q_{i} y\left(t-h_{1, i}\right), \\
\dot{V}_{3, i}\left(x_{t}\right)=y^{T}(t) Q_{i} y(t)-y^{T}\left(t-h_{2, i}\right) Q_{i} y\left(t-h_{2, i}\right), \\
\dot{V}_{4, i}\left(x_{t}\right)=h_{1, i}^{2} \dot{y}^{T}(t) R_{i} \dot{y}(t)-\frac{h_{1, i}}{2} \int_{t-h_{1, i}}^{t} \dot{y}^{T}(s) R_{i} \dot{y}(s) d s-\frac{h_{1, i}}{2} \int_{t-h_{1, i}}^{t} \dot{y}^{T}(s) R_{i} \dot{y}(s) d s, \\
\dot{V}_{5, i}\left(x_{t}\right)=h_{2, i}^{2} \dot{y}^{T}(t) R_{i} \dot{y}(t)-\frac{h_{2, i}}{2} \int_{t-h_{2, i}}^{t} \dot{y}^{T}(s) R_{i} \dot{y}(s) d s-\frac{h_{2, i}}{2} \int_{t-h_{2, i}}^{t} \dot{y}^{T}(s) R_{i} \dot{y}(s) d s, \\
\dot{V}_{6, i}\left(x_{t}\right)=\delta_{i}^{2} \dot{y}^{T}(t) U_{i} \dot{y}(t)-\frac{\delta_{i}}{2} \int_{t-h_{2, i}}^{t-h_{1, i}} \dot{y}^{T}(s) U_{i} \dot{y}(s) d s-\frac{\delta_{i}}{2} \int_{t-h_{2, i}}^{t-h_{1, i}} \dot{y}^{T}(s) R_{i} \dot{y}(s) d s, \\
\dot{V}_{7, i}\left(x_{t}\right)=h_{1, i}^{2} y^{T}(t) Q_{i} y(t)-h_{1, i} \int_{t-h_{1, i}}^{t} x^{T}(s) Y_{i} Q_{i} Y_{i} x(s) d s, \\
\dot{V}_{8, i}\left(x_{t}\right)=h_{2, i}^{2} y^{T}(t) Q_{i} y(t)-h_{2, i} \int_{t-h_{2, i}}^{t} x^{T}(s) Y_{i} Q_{i} Y_{i} x(s) d s .
\end{gathered}
$$


Then by applying Lemma 2.7 and Leibniz-Newton formular, we have

$$
\begin{aligned}
& -\frac{h_{1, i}}{2} \int_{t-h_{1, i}}^{t} \dot{y}^{T}(s) R_{i} \dot{y}(s) d s \\
& \quad \leq y^{T}(t)\left(-\frac{R_{i}}{2}\right) y(t)+y^{T}(t) R_{i} y\left(t-h_{1, i}\right)+y^{T}\left(t-h_{1, i}\right)\left(-\frac{R_{i}}{2}\right) y\left(t-h_{1, i}\right), \\
& -\frac{h_{2, i}}{2} \int_{t-h_{2, i}}^{t} \dot{y}^{T}(s) R_{i} \dot{y}(s) d s \\
& \quad \leq y^{T}(t)\left(-\frac{R_{i}}{2}\right) y(t)+y^{T}(t) R_{i} y\left(t-h_{2, i}\right)+y^{T}\left(t-h_{2, i}\right)\left(-\frac{R_{i}}{2}\right) y\left(t-h_{2, i}\right) .
\end{aligned}
$$

Note that

$$
\begin{aligned}
-\frac{\delta_{i}}{2} \int_{t-h_{2, i}}^{t-h_{1, i}} \dot{y}^{T}(s) U_{i} \dot{y}(s) d s= & -\frac{\delta_{i}}{2} \int_{t-h_{2, i}}^{t-h_{i}(t)} \dot{y}^{T}(s) U_{i} \dot{y}(s) d s-\frac{\delta_{i}}{2} \int_{t-h_{i}(t)}^{t-h_{1, i}} \dot{y}^{T}(s) U_{i} \dot{y}(s) d s \\
= & -\frac{h_{2, i}-h_{i}(t)}{2} \int_{t-h_{2, i}}^{t-h(t)} \dot{y}^{T}(s) U_{i} \dot{y}(s) d s-\frac{h(t)-h_{1, i}}{2} \\
& \times \int_{t-h_{2, i}}^{t-h_{i}(t)} \dot{y}^{T}(s) U_{i} \dot{y}(s) d s-\frac{h_{i}(t)-h_{1, i}}{2} \int_{t-h_{i}(t)}^{t-h_{1, i}} \dot{y}^{T}(s) U_{i} \dot{y}(s) d s \\
& -\frac{h_{2, i}-h_{i}(t)}{2} \int_{t-h(t)}^{t-h_{1, i}} \dot{y}^{T}(s) U_{i} \dot{y}(s) d s .
\end{aligned}
$$

Using Lemma 2.7 yields

$$
\begin{aligned}
& -\frac{h_{2, i}-h_{i}(t)}{2} \int_{t-h_{2, i}}^{t-h_{i}(t)} \dot{y}^{T}(s) U_{i} \dot{y}(s) d s \\
& \quad \leq\left[y\left(t-h_{i}(t)\right)-y\left(t-h_{2, i}\right)\right]^{T}\left(-\frac{U_{i}}{2}\right)\left[y\left(t-h_{i}(t)\right)-y\left(t-h_{2, i}\right)\right] \\
& -\frac{h_{i}(t)-h_{1, i}}{2} \int_{t-h_{i}(t)}^{t-h_{1, i}} \dot{y}^{T}(s) U_{i} \dot{y}(s) d s \\
& \quad \leq\left[y\left(t-h_{1, i}\right)-y\left(t-h_{i}(t)\right)\right]^{T}\left(-\frac{U_{i}}{2}\right)\left[y\left(t-h_{1, i}\right)-y\left(t-h_{i}(t)\right)\right] .
\end{aligned}
$$


Let $\beta=\left(h_{2, i}-h_{i}(t)\right) /\left(h_{2, i}-h_{1, i}\right) \leq 1$. Then

$$
\begin{aligned}
-\frac{h_{2, i}-h_{i}(t)}{2} \int_{t-h_{i}(t)}^{t-h_{1, i}} \dot{y}^{T}(s) U_{i} \dot{y}(s) d s= & -\beta \int_{t-h_{i}(t)}^{t-h_{1, i}}\left(h_{2, i}-h_{1, i}\right) \dot{y}^{T}(s)\left(\frac{U_{i}}{2}\right) \dot{y}(s) d s \\
\leq & -\beta \int_{t-h_{i}(t)}^{t-h_{1, i}}\left(h_{i}(t)-h_{1, i}\right) \dot{y}^{T}(s)\left(\frac{U_{i}}{2}\right) \dot{y}(s) d s \\
\leq & -\beta\left[y\left(t-h_{1, i}\right)-y\left(t-h_{i}(t)\right)\right]^{T}\left(\frac{U_{i}}{2}\right) \\
& \times\left[y\left(t-h_{1, i}\right)-y\left(t-h_{i}(t)\right)\right] . \\
-\frac{h_{i}(t)-h_{1, i}}{2} \int_{t-h_{2, i}}^{t-h_{i}(t)} \dot{y}^{T}(s) U_{i} \dot{y}(s) d s= & -(1-\beta) \int_{t-h_{2, i}}^{t-h_{i}(t)}\left(h_{2, i}-h_{1, i}\right) \dot{y}^{T}(s)\left(\frac{U_{i}}{2}\right) \dot{y}(s) d s \\
\leq & -(1-\beta) \int_{t-h_{2, i}}^{t-h_{i}(t)}\left(h_{2, i}-h_{i}(t)\right) \dot{y}^{T}(s)\left(\frac{U_{i}}{2}\right) \dot{y}(s) d s \\
\leq & -(1-\beta)\left[y\left(t-h_{i}(t)\right)-y\left(t-h_{2, i}\right)\right]^{T}\left(\frac{U_{i}}{2}\right) \\
& \times\left[y\left(t-h_{i}(t)\right)-y\left(t-h_{2, i}\right)\right] .
\end{aligned}
$$

Therefore from (3.18)-(3.19), we have

$$
\begin{aligned}
-\frac{\delta_{i}}{2} \int_{t-h_{2, i}}^{t-h_{1, i}} \dot{y}^{T}(s) U_{i} \dot{y}(s) d s \leq & {\left[y\left(t-h_{i}(t)\right)-y\left(t-h_{2, i}\right)\right]^{T}\left(-\frac{U_{i}}{2}\right)\left[y\left(t-h_{i}(t)\right)-y\left(t-h_{2, i}\right)\right] } \\
& +\left[y\left(t-h_{i}(t)\right)-y\left(t-h_{2, i}\right)\right]^{T}\left(-\frac{U_{i}}{2}\right)\left[y\left(t-h_{i}(t)\right)-y\left(t-h_{2, i}\right)\right] \\
& -\beta\left[y\left(t-h_{1, i}\right)-y\left(t-h_{i}(t)\right)\right]^{T}\left(\frac{U_{i}}{2}\right)\left[y\left(t-h_{1, i}\right)-y\left(t-h_{i}(t)\right)\right] \\
& -(1-\beta)\left[y\left(t-h_{i}(t)\right)-y\left(t-h_{2, i}\right)\right]^{T}\left(\frac{U_{i}}{2}\right) \\
& \times\left[y\left(t-h_{i}(t)\right)-y\left(t-h_{2, i}\right)\right] .
\end{aligned}
$$

Furthermore, from the following zero equation

$$
-P_{i} \dot{y}(t)+A_{i} P_{i} y(t)+D_{i} P_{i} y\left(t-h_{i}(t)\right)-0.5 B_{i} B_{i}^{T} y(t)=0,
$$

we obtain

$$
-2 \dot{y}^{T} P_{i} \dot{y}(t)+2 \dot{y}^{T} A_{i} P_{i} y(t)+2 \dot{y}^{T} D_{i} P_{i} y\left(t-h_{i}(t)\right)-2 \dot{y}^{T} 0.5 B_{i} B_{i}^{T} y(t)=0 .
$$


Hence, from (3.5),(3.8)-(3.16),(3.20), and (3.22), we can get

$$
\begin{aligned}
\dot{V}_{i}\left(x_{t}\right) \leq & \xi^{T}(t) \Omega_{i} \xi(t)-\beta\left[y\left(t-h_{1, i}\right)-y(t-h(t))\right]^{T}\left(\frac{U_{i}}{2}\right)\left[y\left(t-h_{1, i}\right)-y\left(t-h_{i}(t)\right)\right] \\
& -(1-\beta)\left[y\left(t-h_{i}(t)\right)-y\left(t-h_{2, i}\right)\right]^{T}\left(\frac{U_{i}}{2}\right)\left[y\left(t-h_{i}(t)\right)-y\left(t-h_{2, i}\right)\right] \\
& -\frac{h_{1, i}}{2} \int_{t-h_{1, i}}^{t} \dot{y}^{T}(s) R_{i} \dot{y}(s) d s-\frac{h_{2, i}}{2} \int_{t-h_{2, i}}^{t} \dot{y}^{T}(s) R_{i} \dot{y}(s) d s \\
& -\frac{\delta_{i}}{2} \int_{t-h_{2, i}}^{t-h_{1, i}} \dot{y}^{T}(s) R_{i} \dot{y}(s) d s-h_{1, i} \int_{t-h_{1, i}}^{t} x^{T}(s) Y_{i} Q_{i} Y_{i} x(s) d s \\
& -h_{2, i} \int_{t-h_{2, i}}^{t} x^{T}(s) Y_{i} Q_{i} Y_{i} x(s) d s \\
= & \xi^{T}(t)\left[(1-\beta) \Omega_{1, i}+\beta \Omega_{2, i}\right] \xi(t)-\frac{h_{1, i}}{2} \int_{t-h_{1, i}}^{t} \dot{y}^{T}(s) R_{i} \dot{y}(s) d s \\
& -\frac{h_{2, i}}{2} \int_{t-h_{2, i}}^{t} \dot{y}^{T}(s) R_{i} \dot{y}(s) d s-\frac{\delta_{i}}{2} \int_{t-h_{2, i}}^{t-h_{1, i}} \dot{y}^{T}(s) R_{i} \dot{y}(s) d s, \\
& -h_{1, i} \int_{t-h_{1, i}}^{t} x^{T}(s) Y_{i} Q_{i} Y_{i} x(s) d s-h_{2, i} \int_{t-h_{2, i}}^{t} x^{T}(s) Y_{i} Q_{i} Y_{i} x(s) d s,
\end{aligned}
$$

where $\xi^{T}(t)=\left[y^{T}(t) \dot{y}^{T}(t) y^{T}\left(t-h_{1, i}\right) y^{T}\left(t-h_{2, i}\right) y^{T}\left(t-h_{i}(t)\right)\right]$.

Suppose $\tau$ is the time when the system switches from state $j$ to state $i$, that is $I\left(\tau^{+}\right)=$ $i$ and $I\left(\tau^{-}\right)=j$, where $\tau^{+}$and $\tau^{-}$are the right and the left limit of the time $\tau$, respectively. According to Lemma 2.6, we obtain

$$
\begin{aligned}
V_{1, i}\left(x_{\tau^{+}}\right) & =x^{T}(\tau) Y_{i} x(\tau) \\
& \leq \frac{\lambda_{1}}{\lambda_{2}} \lambda_{2} x^{T}(\tau) x(\tau) \\
& \leq \frac{\lambda_{1}}{\lambda_{2}} x^{T}(\tau) P_{j} x(\tau) \\
& =\frac{\lambda_{1}}{\lambda_{2}} V_{1, j}\left(x_{\tau^{-}}\right) .
\end{aligned}
$$

We can apply this argument to integral terms in the Lypunov-Krasovskii function, so we get

$$
V_{i}\left(x_{\tau^{+}}\right) \leq \frac{\lambda_{1}}{\lambda_{2}} V_{j}\left(x_{\tau^{-}}\right)
$$

Now let $v$ be the time when the system switches from state $k$ to state $j$, that is, $I\left(v^{+}\right)=j$ and $I\left(v^{-}\right)=k$, where $v^{+}$and $v^{-}$are a right and a left limit of the time $v$, respectively. In order 
to show that the switched system is stable, we need to compare $V_{i}\left(x_{\tau^{+}}\right)$with $V_{j}\left(x_{v^{+}}\right)$and estimate the upper bound for the term $\xi^{T}(t)\left[(1-\beta) \Omega_{1, i}+\beta \Omega_{2, i}\right] \xi(t)$ in the inequality (3.23). Hence we consider two following possible cases.

Case $1\left(0 \leq h_{1} \leq h_{1, i} \leq h(t) \leq h_{a, i} \leq h_{2, i} \leq h_{2}\right)$. Since $0 \leq \beta \leq 1, \Omega_{1, i}<0$ and $\Omega_{2, i}<0$, we have

$$
\begin{aligned}
\xi^{T}(t) & {\left[(1-\beta) \Omega_{1, i}+\beta \Omega_{2, i}\right] \xi(t) } \\
& \leq \xi^{T}(t)\left[\beta \Omega_{2, i}\right] \xi(t) \\
& \leq-\left(\frac{h_{2, i}-h_{a, i}}{\delta_{i}}\right) \lambda_{5}\left(\|y(t)\|^{2}+\|\dot{y}(t)\|^{2}+\left\|y\left(t-h_{1, i}\right)\right\|^{2}\right. \\
& \left.\quad+\left\|y\left(t-h_{2, i}\right)\right\|^{2}+\left\|y\left(t-h_{i}(t)\right)\right\|^{2}\right) \\
& \leq-\left(\frac{h_{2, i}-h_{a, i}}{\delta_{i}}\right) \lambda_{5}\left(\|y(t)\|^{2}\right) \\
& =-\left(\frac{h_{2, i}-h_{a, i}}{\delta_{i}}\right) \frac{\lambda_{5} \lambda_{3}}{\lambda_{3}}\left(\|y(t)\|^{2}\right) \\
& \leq-\left(\frac{h_{2, i}-h_{a, i}}{\delta}\right) \frac{\lambda_{5}}{\lambda_{3}} x^{T}(t) P_{i}^{-1} P_{i} P_{i}^{-1} x(t) \\
& \leq-\left(\frac{h_{2, i}-h_{a, i}}{\delta}\right) \frac{\lambda_{5}}{\lambda_{3}} V_{1, i} \\
& \leq-\left(\frac{h_{2, i}-h_{a, i}}{\delta}\right) \frac{\lambda^{*}}{\lambda_{3}} V_{1, i} \\
& \leq-\left(\frac{h^{*}}{\delta}\right) \frac{\lambda^{*}}{\lambda_{3}} V_{1, i} .
\end{aligned}
$$

Case $2\left(0 \leq h_{1} \leq h_{1, i} \leq h_{a, i} \leq h(t) \leq h_{2, i} \leq h_{2}\right)$. Since $0 \leq \beta \leq 1, \Omega_{1, i}<0$ and $\Omega_{2, i}<0$, we get

$$
\begin{aligned}
& \xi^{T}(t)\left[(1-\beta) \Omega_{1, i}+\beta \Omega_{2, i}\right] \xi(t) \\
& \leq \xi^{T}(t)\left[(1-\beta) \Omega_{1, i}\right] \xi(t) \\
& \leq-\left(\frac{h_{a, i}-h_{1, i}}{\delta_{i}}\right) \lambda_{4}\left(\|y(t)\|^{2}+\|\dot{y}(t)\|^{2}+\left\|y\left(t-h_{1, i}\right)\right\|^{2}\right. \\
& \left.\quad+\left\|y\left(t-h_{2, i}\right)\right\|^{2}+\left\|y\left(t-h_{i}(t)\right)\right\|^{2}\right) \\
& \leq-\left(\frac{h_{a, i}-h_{1, i}}{\delta_{i}}\right) \lambda_{4}\left(\|y(t)\|^{2}\right)
\end{aligned}
$$


Journal of Applied Mathematics

$$
\begin{aligned}
& =-\left(\frac{h_{a, i}-h_{1, i}}{\delta_{i}}\right) \frac{\lambda_{4} \lambda_{3}}{\lambda_{3}}\left(\|y(t)\|^{2}\right) \\
& \leq-\left(\frac{h_{a, i}-h_{1, i}}{\delta}\right) \frac{\lambda_{4}}{\lambda_{3}} x^{T}(t) P_{i}^{-1} P_{i} P_{i}^{-1} x(t) \\
& \leq-\left(\frac{h_{a, i}-h_{1, i}}{\delta}\right) \frac{\lambda_{4}}{\lambda_{3}} V_{1, i} .
\end{aligned}
$$

Note that $h_{2, i}-h_{a, i}=h_{a, i}-h_{1, i}$, so we obtain

$$
\begin{aligned}
\xi^{T}(t)\left[(1-\beta) \Omega_{1, i}+\beta \Omega_{2, i}\right] \xi(t) & \leq-\left(\frac{h_{2, i}-h_{a, i}}{\delta}\right) \frac{\lambda^{*}}{\lambda_{3}} V_{1, i} \\
& \leq-\left(\frac{h^{*}}{\delta}\right) \frac{\lambda^{*}}{\lambda_{3}} V_{1, i} .
\end{aligned}
$$

Moreover, from the definition of $V_{2, i}, \ldots, V_{8, i}$, we can get

$$
\begin{aligned}
-\frac{h_{1, i}}{2} \int_{t-h_{1, i}}^{t} \dot{y}^{T}(s) R_{i} \dot{y}(s) d s & \leq-\frac{1}{2} \int_{-h_{1, i}}^{0} \int_{t+s}^{t} \dot{x}^{T}(\theta) Y_{i} R_{i} Y_{i} \dot{x}(\theta) d \theta d s \\
& \leq-\frac{1}{2 h_{2, i}} V_{4, i} \\
-\frac{h_{2, i}}{2} \int_{t-h_{2, i}}^{t} \dot{y}^{T}(s) R_{i} \dot{y}(s) d s & \leq-\frac{1}{2} \int_{-h_{2, i}}^{0} \int_{t+s}^{t} \dot{x}^{T}(\theta) Y_{i} R_{i} Y_{i} \dot{x}(\theta) d \theta d s \\
& \leq-\frac{1}{2 h_{1, i}} V_{5, i}, \\
-\frac{\delta_{i}}{2} \int_{t-h_{2, i}}^{t-h_{1, i}} \dot{y}^{T}(s) U_{i} \dot{y}(s) d s & \leq-\frac{1}{2} \int_{-h_{2, i}}^{-h_{1, i}} \int_{t+s}^{t} \dot{x}^{T}(\theta) Y_{i} U_{i} Y_{i} \dot{x}(\theta) d \theta d s \\
& \leq-\frac{1}{2 \delta_{i}} V_{6, i} .
\end{aligned}
$$

Since

$$
\begin{aligned}
-h_{1, i} \int_{t-h_{1, i}}^{t} x^{T}(s) Y_{i} Q_{i} Y_{i} x(s) d s & \leq-\int_{-h_{1, i}}^{0} \int_{t+s}^{t} x^{T}(\theta) Y_{i} Q_{i} Y_{i} x(\theta) d \theta d s \\
& \leq-\frac{1}{h_{1, i}} V_{7, i},
\end{aligned}
$$


we can get

$$
\begin{gathered}
-h_{1, i} \int_{t-h_{1, i}}^{t} x^{T}(s) Y_{i} Q_{i} Y_{i} x(s) d s-h_{1, i} \int_{t-h_{1, i}}^{t} x^{T}(s) Y_{i} Q_{i} Y_{i} x(s) d s \\
\leq-\frac{1}{h_{1, i}} V_{7, i}-h_{1, i} \int_{t-h_{1, i}}^{t} x^{T}(s) Y_{i} Q_{i} Y_{i} x(s) d s .
\end{gathered}
$$

So we obtain

$$
-h_{1, i} \int_{t-h_{1, i}}^{t} x^{T}(s) Y_{i} Q_{i} Y_{i} x(s) d s \leq-\frac{1}{2 h_{1, i}} V_{7, i}-\frac{1}{2} V_{2, i} .
$$

Similar to (3.32), we have

$$
-h_{2, i} \int_{t-h_{2, i}}^{t} x^{T}(s) Y_{i} Q_{i} Y_{i} x(s) d s \leq-\frac{1}{2 h_{2, i}} V_{3, i}-\frac{1}{2} V_{8, i} .
$$

According to (3.23), (3.28)-(3.33), we have

$$
\begin{aligned}
\dot{V}_{i}\left(x_{t}\right) & \leq-\min \left\{\frac{h^{*} \lambda^{*}}{\delta \lambda_{3}}, \frac{1}{2 h_{1, i}}, \frac{1}{2 h_{2, i}}, \frac{1}{2 \delta_{i}}, \frac{1}{2}\right\} V_{i}\left(x_{t}\right) \\
& \leq-\min \left\{\frac{h^{*} \lambda^{*}}{\delta \lambda_{3}}, \frac{1}{2 h_{2}}, \frac{1}{2}\right\} V_{i}\left(x_{t}\right) \\
& =-\rho V_{i}\left(x_{t}\right) .
\end{aligned}
$$

As a result, we get

$$
\dot{V}_{j}\left(x_{t}\right) \leq-\rho V_{j}\left(x_{t}\right),
$$

which yields

$$
\frac{1}{V_{j}\left(x_{t}\right)} d V_{j}\left(x_{t}\right) \leq-\rho d t
$$

Integrating (3.36) on $(v, \tau)$, we obtain

$$
V_{j}\left(x_{\tau^{-}}\right) \leq V_{j}\left(x_{v^{+}}\right) e^{-\rho(\tau-v)}
$$

From (3.25) and (3.37), we have

$$
V_{i}\left(x_{\tau^{+}}\right) \leq \frac{\lambda_{1}}{\lambda_{2}} V_{j}\left(x_{v^{+}}\right) e^{-\rho(\tau-v)}
$$


Since

$$
\tau-v \geq T_{0} \geq \frac{1}{\rho} \ln \left(\frac{\lambda_{1}}{\alpha \lambda_{2}}\right)
$$

we obtain from (3.38) that

$$
V_{i}\left(x_{\tau^{+}}\right) \leq \alpha V_{j}\left(x_{v^{+}}\right) .
$$

Let $N(t)$ be the number of time the system is switched on $[0, t)$. Since the switched system (3.1) undergoes infinite times of switches on $[0, \infty)$, we obtain $\lim _{t \rightarrow \infty} N(t)=\infty$. Assume that $I(t)=i$ and $I(0)=i_{0}$. Then, according to (3.7) and (3.40), we have

$$
c_{1}\|x(t)\|^{2} \leq V_{i}\left(x_{t}\right) \leq \alpha^{N(t)} V_{i_{0}}\left(x_{0}\right) .
$$

Since $0<\alpha<1$ and $\lim _{t \rightarrow \infty} N(t)=\infty$, it follows from (3.41) that $\|x(t)\| \rightarrow 0$ as $t \rightarrow \infty$. We conclude that the zero solution of (3.1) is stabilizable.

\subsection{Robust Stabilization for Switched Systems with Interval Time-Varying Delay}

Theorem 3.2. Given $\alpha \in(0,1)$. If there exists symmetric positive definite matrices $P_{i}, Q_{i}, R_{i}, U_{i}$ such that the following conditions hold:

$$
\begin{aligned}
& \Omega_{1, i}=\Omega_{i}-\left[\begin{array}{ll}
000 & -I I
\end{array}\right]^{T} U_{i}\left[\begin{array}{ll}
000 & -I I
\end{array}\right]<0, \\
& \Omega_{2, i}=\Omega_{i}-\left[\begin{array}{ll}
00 I 0 & -I
\end{array}\right]^{T} U_{i}\left[\begin{array}{ll}
00 I 0 & -I
\end{array}\right]<0, \\
& \Omega_{3, i}=\left[\begin{array}{ccccc}
\Omega_{311, i} & P_{i} H_{1, i}^{T} & P_{i} H_{1, i}^{T} & \frac{B_{i} H_{3, i}^{T}}{2} & \frac{B_{i} H_{3, i}^{T}}{2} \\
* & -\epsilon_{1} I & 0 & 0 & 0 \\
* & * & -\epsilon_{2} I & 0 & 0 \\
* & * & * & \frac{-\epsilon_{5} I}{2} & 0 \\
* & * & * & * & \frac{-\epsilon_{6} I}{2}
\end{array}\right]<0, \\
& \Omega_{4, i}=\left[\begin{array}{ccc}
\Omega_{411, i} & P_{i} H_{2, i}^{T} & P_{i} H_{2, i}^{T} \\
* & -\epsilon_{3} I & 0 \\
* & * & -\epsilon_{4} I
\end{array}\right]<0, \\
& \Omega_{i}=\left[\begin{array}{ccccc}
\Lambda_{11, i} & \Lambda_{12, i} & \Lambda_{13, i} & \Lambda_{14, i} & \Lambda_{15, i} \\
* & \Lambda_{22, i} & 0 & 0 & \Lambda_{25, i} \\
* & * & \Lambda_{33, i} & 0 & \Lambda_{35, i} \\
* & * & * & \Lambda_{44, i} & \Lambda_{45, i} \\
* & * & * & * & \Lambda_{55, i}
\end{array}\right],
\end{aligned}
$$


where

$$
\begin{aligned}
& \Lambda_{11, i}=P_{i} A_{i}^{T}+A_{i} P_{i}-0.5 B_{i} B_{i}^{T}+\left(2+h_{1, i}^{2}+h_{2, i}^{2}\right) Q_{i}-0.5 R_{i}+\epsilon_{1} E_{1}^{T} E_{1}+\epsilon_{3} E_{2}^{T} E_{2}+\frac{\epsilon_{5}}{2} E_{3}^{T} E_{3} ; \\
& \Lambda_{12, i}=P_{i} A_{i}^{T}-0.5 B_{i} B_{i}^{T} ; \\
& \Lambda_{13, i}=0.5 R_{i} ; \\
& \Lambda_{14, i}=0.5 R_{i} ; \\
& \Lambda_{15, i}=D_{i} P_{i} ; \\
& \Lambda_{22, i}=\left(h_{1, i}^{2}+h_{2, i}^{2}+\delta_{i}^{2}\right) Q_{i}-2 R_{i}+\epsilon_{2} E_{1}^{T} E_{1}+\epsilon_{4} E_{2}^{T} E_{2}+\frac{\epsilon_{6}}{2} E_{3}^{T} E_{3} ; \\
& \Lambda_{25, i}=D_{i} P_{i} ; \\
& \Lambda_{33, i}=-Q_{i}-0.5 R_{i}-0.5 U_{i} ; \\
& \Lambda_{35, i}=0.5 U_{i} ; \\
& \Lambda_{44, i}=-Q_{i}-0.5 R_{i}-0.5 U_{i} ; \\
& \Lambda_{45, i}=0.5 U_{i} ; \\
& \Lambda_{55, i}=-0.5 U_{i} ; \\
& \Omega_{311, i}=-0.5 B_{i} B_{i}^{T}-0.5 R_{i} ; \\
& \Omega_{411, i}=-0.5 U_{i} .
\end{aligned}
$$

Then for any switching rule satisfying (3.3) the switched system (2.1) is robustly stabilizable under the feedback controller given by (3.4).

Proof. Construct Lyapunov-Krasovskii functional as in (3.5), we can proof this theorem by using a similar argument as in the proof of Theorem 3.1. By replacing $A_{i}, D_{i}, B_{i}$ in (3.8) with $A_{i}+E_{1, i} F_{1, i}(t) H_{1, i}, D_{i}+E_{2, i} F_{2, i}(t) H_{2, i}, B_{i}+E_{3, i} F_{3, i}(t) H_{3, i}$, respectively, we obtain

$$
\begin{aligned}
\dot{V}_{i}\left(x_{t}\right) \leq & y^{T}(t)\left[P_{i}\left(A_{i}+E_{1, i} F_{1, i}(t) H_{1, i}\right)^{T}+\left(A_{i}+E_{1, i} F_{1, i}(t) H_{1, i}\right) P_{i}\right] y(t) \\
& +2 y^{T}(t)\left(D_{i}+E_{2, i} F_{2, i}(t) H_{2, i}\right) P_{i} y\left(t-h_{i}(t)\right)-y^{T}(t) B_{i}^{T} B_{i} y(t) \\
& -y^{T}(t) E_{3, i} F_{3, i}(t) H_{3, i} B_{i}^{T} y(t)+y^{T}(t)\left(2+h_{1, i}^{2}+h_{2, i}^{2}\right) Q_{i} y(t) \\
& -y^{T}\left(t-h_{1}\right) Q_{i} y\left(t-h_{1}\right)-y^{T}\left(t-h_{2}\right) Q_{i} y\left(t-h_{2}\right) \\
& +\dot{y}^{T}(t)\left[\left(h_{1, i}^{2}+h_{1, i}^{2}\right) R_{i}+\delta_{i}^{2} U_{i}\right] \dot{y}(t)+y^{T}(t)\left(-\frac{R_{i}}{2}\right) y(t) \\
& +y^{T}(t) R_{i} y\left(t-h_{1, i}\right)+y^{T}\left(t-h_{1, i}\right)\left(-\frac{R_{i}}{2}\right) y\left(t-h_{1, i}\right)
\end{aligned}
$$


Journal of Applied Mathematics

$$
\begin{aligned}
& +y^{T}(t)\left(-\frac{R_{i}}{2}\right) y(t)+y^{T}(t) R_{i} y\left(t-h_{2, i}\right)+y^{T}\left(t-h_{2, i}\right)\left(-\frac{R_{i}}{2}\right) y\left(t-h_{2, i}\right) \\
& +\left[y\left(t-h_{i}(t)\right)-y\left(t-h_{2, i}\right)\right]^{T}\left(-\frac{U_{i}}{2}\right)\left[y\left(t-h_{i}(t)\right)-y\left(t-h_{2, i}\right)\right] \\
& +\left[y\left(t-h_{i}(t)\right)-y\left(t-h_{2, i}\right)\right]^{T}\left(-\frac{U_{i}}{2}\right)\left[y\left(t-h_{i}(t)\right)-y\left(t-h_{2, i}\right)\right] \\
& -\beta\left[y\left(t-h_{1, i}\right)-y\left(t-h_{i}(t)\right)\right]^{T}\left(-\frac{U_{i}}{2}\right)\left[y\left(t-h_{1, i}\right)-y\left(t-h_{i}(t)\right)\right] \\
& -(1-\beta)\left[y\left(t-h_{i}(t)\right)-y\left(t-h_{2, i}\right)\right]^{T}\left(-\frac{U_{i}}{2}\right)\left[y\left(t-h_{i}(t)\right)-y\left(t-h_{2, i}\right)\right] \\
& -2 \dot{y}^{T} P_{i} \dot{y}(t)+2 \dot{y}^{T}\left(A_{i}+E_{1, i} F_{1, i}(t) H_{1, i}\right) P_{i} y(t)+2 \dot{y}^{T}\left(D_{i}+E_{2, i} F_{2, i}(t) H_{2, i}\right) P_{i} \\
& \times y\left(t-h_{i}(t)\right)-2 \dot{y}^{T} 0.5 B_{i} B_{i}^{T} y(t)-\dot{y}^{T} E_{3, i} F_{3, i}(t) H_{3, i} B_{i}^{T} y(t) \\
& -\frac{h_{1, i}}{2} \int_{t-h_{1, i}}^{t} \dot{y}^{T}(s) R_{i} \dot{y}(s) d s-\frac{h_{2, i}}{2} \int_{t-h_{2, i}}^{t} \dot{y}^{T}(s) R_{i} \dot{y}(s) d s \\
& -\frac{\delta_{i}}{2} \int_{t-h_{2, i}}^{t-h_{1, i}} \dot{y}^{T}(s) R_{i} \dot{y}(s) d s-h_{1, i} \int_{t-h_{1, i}}^{t} x^{T}(s) Y_{i} Q_{i} Y_{i} x(s) d s \\
& -h_{2, i} \int_{t-h_{2, i}}^{t} x^{T}(s) Y_{i} Q_{i} Y_{i} x(s) d s .
\end{aligned}
$$

And using Lemma 2.5, we can get the following upper bounds for the uncertain terms in (3.48):

$$
\begin{aligned}
2 y^{T}(t) E_{1, i} F_{1, i}(t) H_{1, i} P_{i} y(t) \leq & \epsilon_{1, i} y^{T}(t) E_{1, i}^{T} E_{1, i} y(t)+\epsilon_{1, i}^{-1} y^{T}(t) P_{i} H_{1, i}^{T} H_{1, i} P_{i} y(t) \\
2 \dot{y}^{T}(t) E_{1, i} F_{1, i}(t) H_{1, i} P_{i} y(t) \leq & \epsilon_{2, i} \dot{y}^{T}(t) E_{1, i}^{T} E_{1, i} \dot{y}(t)+\epsilon_{2, i}^{-1} y^{T}(t) P_{i} H_{1, i}^{T} H_{1, i} P_{i} y(t) \\
2 y^{T}(t) E_{2, i} F_{2, i}(t) H_{2, i} P_{i} y\left(t-h_{i}(t)\right) \leq & \epsilon_{3, i} y^{T}(t) E_{2, i}^{T} E_{2, i} y(t) \\
& +\epsilon_{3, i}^{-1} y^{T}\left(t-h_{i}(t)\right) P_{i} H_{2, i}^{T} H_{2, i} P_{i} y\left(t-h_{i}(t)\right) \\
2 \dot{y}^{T}(t) E_{2, i} F_{2, i}(t) H_{2, i} P_{i} y\left(t-h_{i}(t)\right) \leq & \epsilon_{4, i} \dot{y}^{T}(t) E_{2, i}^{T} E_{2, i} \dot{y}(t) \\
& +\epsilon_{4, i}^{-1} y^{T}\left(t-h_{i}(t)\right) P_{i} H_{2, i}^{T} H_{2, i} P_{i} y\left(t-h_{i}(t)\right) \\
-y^{T}(t) E_{3, i} F_{3, i}(t) H_{3, i} B_{i}^{T} y(t) \leq \frac{\epsilon_{5, i}}{2} y^{T}(t) E_{3, i}^{T} E_{3, i} y(t)+\frac{\epsilon_{5, i}^{-1}}{2} y^{T}(t) B_{i} H_{3, i}^{T} H_{3, i} B_{i} y(t) & \\
-\dot{y}^{T}(t) E_{3, i} F_{3, i}(t) H_{3, i} B_{i}^{T} y(t) \leq & \frac{\epsilon_{6, i}}{2} \dot{y}^{T}(t) E_{3, i}^{T} E_{3, i} \dot{y}(t)+\frac{\epsilon_{6, i}^{-1}}{2} y^{T}(t) B_{i} H_{3, i}^{T} H_{3, i} B_{i} y(t)
\end{aligned}
$$


According to (3.48)-(3.49), it follows that

$$
\begin{aligned}
\dot{V}_{i}\left(x_{t}\right) \leq & \xi^{T}(t)\left[(1-\beta) \Omega_{1, i}+\beta \Omega_{2, i}\right] \xi(t)+y^{T}(t) \Psi_{3, i} y(t)+y^{T}\left(t-h_{i}(t)\right) \Psi_{4, i} y\left(t-h_{i}(t)\right) \\
& -\frac{h_{1, i}}{2} \int_{t-h_{1, i}}^{t} \dot{y}^{T}(s) R_{i} \dot{y}(s) d s-\frac{h_{2, i}}{2} \int_{t-h_{2, i}}^{t} \dot{y}^{T}(s) R_{i} \dot{y}(s) d s \\
& -\frac{\delta_{i}}{2} \int_{t-h_{2, i}}^{t-h_{1, i}} \dot{y}^{T}(s) R_{i} \dot{y}(s) d s,-h_{1, i} \int_{t-h_{1, i}}^{t} x^{T}(s) Y_{i} Q_{i} Y_{i} x(s) d s \\
& -h_{2, i} \int_{t-h_{2, i}}^{t} x^{T}(s) Y_{i} Q_{i} Y_{i} x(s) d s,
\end{aligned}
$$

where

$$
\begin{aligned}
& \Psi_{3, i}=\Omega_{311, i}+\varepsilon_{1, i}^{-1} P_{i} H_{1, i}^{T} H_{1, i} P_{i}+\epsilon_{2, i}^{-1} P_{i} H_{1, i}^{T} H_{1, i} P_{i}+\frac{\epsilon_{5, i}^{-1}}{2} B_{i} H_{3, i}^{T} H_{3, i} B_{i}^{T}+\frac{\epsilon_{6, i}^{-1}}{2} B_{i} H_{3, i}^{T} H_{3, i} B_{i}^{T}, \\
& \Psi_{4, i}=\Omega_{311, i}+\epsilon_{3, i}^{-1} P_{i} H_{2, i}^{T} H_{2, i} P_{i}+\epsilon_{4, i}^{-1} P_{i} H_{2, i}^{T} H_{2, i} P_{i} .
\end{aligned}
$$

Applying Lemma 2.4, the LMIs $\Psi_{3, i}<0$ and $\Psi_{4, i}<0$ are equivalent to $\Omega_{3, i}<0$ and $\Omega_{4, i}<0$, respectively. Similarly to the previous theorem we can conclude that the switched system (2.1) is robustly stabilizable.

Remark 3.3. Our proposed method can remove the conservative restrictions on the derivatives of the time-varying delays, meanwhile traditional design methods require the condition $\dot{h}(t) \leq h_{D}$ (see $\left.[14,19,20]\right)$. So our method can deal with fast time-varying delays. Moreover, the improved method need not to introduce any free-weighting matrix variables which turn out to be less conservative than results in $[14,20,21]$.

\section{Numerical Examples}

In this section, we provide some examples to illustrate the effectiveness of our results in Theorems 3.1 and 3.2.

Example 4.1. Consider the nominal switched systems with interval time-varying delay

$$
\dot{x}(t)=A_{i} x(t)+D_{i} x\left(t-h_{i}(t)\right)+B_{i} u_{i}(t), \quad i=1,2,
$$

with

$$
\begin{array}{lll}
A_{1}=\left[\begin{array}{cc}
-2 & 0 \\
0 & 0.5
\end{array}\right], & D_{1}=\left[\begin{array}{cc}
-0.2 & 0.1 \\
0.1 & -0.1
\end{array}\right], & B_{1}=\left[\begin{array}{l}
0 \\
1
\end{array}\right], \\
A_{2}=\left[\begin{array}{cc}
-3 & 1 \\
0 & 0.1
\end{array}\right], & D_{2}=\left[\begin{array}{cc}
-0.1 & 0.1 \\
0.1 & -0.1
\end{array}\right], & B_{2}=\left[\begin{array}{l}
0 \\
1
\end{array}\right] .
\end{array}
$$


Table 1: Maximum allowable upper bounds $h_{2}$ of the time-varying delay for different values of the lower bounds $h_{1}$ in example 1 of [8].

\begin{tabular}{lcccc}
\hline Result & $h_{1}$ & & & $h_{2}$ \\
& & {$[8]$} & {$[4]$} & $\begin{array}{c}\text { Our results } \\
\text { No restriction on } \dot{h}(t)\end{array}$ \\
\hline$h_{d}=0.4$ & 0 & 1.2644 & - & 1.2843 \\
$h_{d} \geq 1$ or unknown & 0 & 0.9339 & - & 1.2843 \\
$h_{d} \geq 1$ or unknown & 0.3 & - & 0.9514 & 1.3120 \\
$h_{d} \geq 1$ or unknown & 0.6 & - & 1.04 & 1.3913 \\
\hline
\end{tabular}

And we choose $\alpha=0.9, h_{1}(t)=0.1+|\sin t|, h_{2}(t)=0.1+|\cos t|$, so we get $h_{1}=0.1, h_{2}=1.1$. Then, by using the LMI control toolbox in Matlab, solutions of LMIs (3.2) are given by

$$
\begin{aligned}
& P_{1}=\left[\begin{array}{cc}
0.8273 & -0.0052 \\
-0.0052 & 0.8167
\end{array}\right], \quad Q_{1}=\left[\begin{array}{cc}
0.2445 & 0.0040 \\
0.0040 & 0.0665
\end{array}\right], \quad R_{1}=\left[\begin{array}{cc}
0.1238 & 0.0030 \\
0.0030 & 0.5581
\end{array}\right], \\
& U_{1}=\left[\begin{array}{cc}
0.2249 & -0.0084 \\
-0.0084 & 0.3873
\end{array}\right], \quad P_{2}=\left[\begin{array}{ll}
3.4735 & 1.2444 \\
1.2444 & 4.2337
\end{array}\right], \quad Q_{2}=\left[\begin{array}{cc}
0.7132 & 0.1563 \\
0.1563 & 0.1691
\end{array}\right], \\
& R_{2}=\left[\begin{array}{ll}
0.4429 & 0.9777 \\
0.9777 & 2.9654
\end{array}\right], \quad U_{2}=\left[\begin{array}{ll}
0.5684 & 0.4259 \\
0.4259 & 1.8023
\end{array}\right] \text {, }
\end{aligned}
$$

with stabilizing controllers

$$
u_{1}(t)=\left[\begin{array}{ll}
-0.0039 & -0.6122
\end{array}\right] x(t), \quad u_{2}(t)=[0.0473-0.1320] x(t) .
$$

By computation, we obtain $\lambda^{*}=0.0461, \lambda_{1}=1.2276, \lambda_{2}=0.1940, \lambda_{3}=5.1547, h^{*}=0.5$. Thus, $\rho=0.0054$ and from (3.3) we obtain

$$
T_{0} \geq 361.1694
$$

Hence, from Theorem 3.1, the switched systems (4.1) with arbitrary switching law subject to (4.5) are stabilizable under the feedback controllers which are shown in (4.4).

By choosing initial condition as $\phi(t)=\left[\begin{array}{c}\sin (t) \\ \sin (t)\end{array}\right], t \in[-1.1,0]$, the trajectories of solutions of the switched system and the trajectories of solutions of subsystems 1 and 2 for this example are shown in Figures 1, 2, 3, and 4.

In Tables 1 and 2 we give comparison of maximum allowable value of $h_{2}$ of asymptotic stability of nominal switched systems obtained in [14, 22] and Theorem 3.1. As we can see that for some of $h_{1}$, the maximum allowable bounds for $h_{2}$ obtained from Theorem 3.1 are greater than that obtained in $[14,22]$. More important, the differentiability of the time delay $h(t)$ is not required in our theorem.

Example 4.2. Consider the following uncertain switched systems with interval time-varying delay

$$
\dot{x}(t)=\left(A_{i}+\Delta A_{i}\right) x(t)+\left(D_{i}+\Delta D_{i}\right) x\left(t-h_{i}(t)\right)+\left(B_{i}+\Delta B_{i}\right) u_{i}(t), \quad i=1,2,
$$




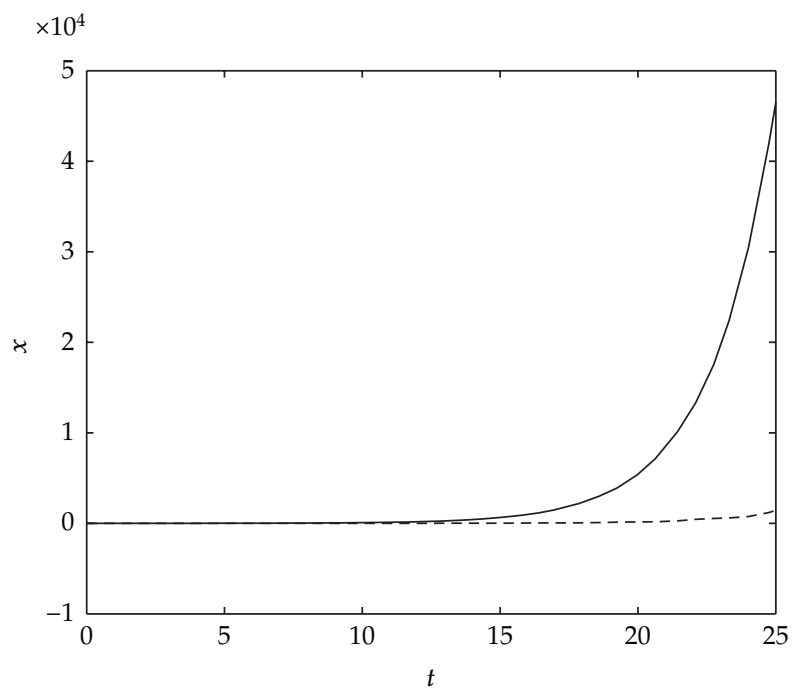

$---x_{1}$

$-x_{2}$

Figure 1: The trajectory of solution of system $i=1$.

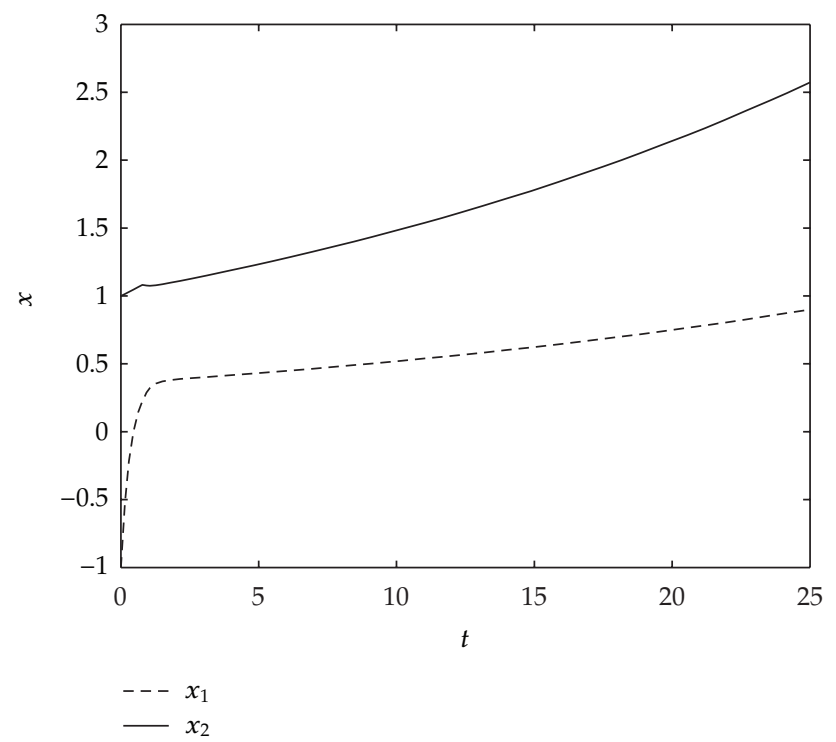

Figure 2: The trajectory of solution of system $i=2$.

Table 2: Maximum allowable upper bounds $h_{2}$ of the time-varying delay for different values of the lower bounds $h_{1}$ in example 2 of [4].

\begin{tabular}{lccc}
\hline Result & $h_{1}$ & \multicolumn{2}{c}{$h_{2}$} \\
& & {$[4]$} & $\begin{array}{c}\text { Our results } \\
\text { No restriction on } \dot{h}(t)\end{array}$ \\
\hline$h_{d} \geq 1$ or unknown & 0 & 0.687 & 0.9871 \\
$h_{d} \geq 1$ or unknown & 0.4 & 0.856 & 1.0227 \\
\hline
\end{tabular}




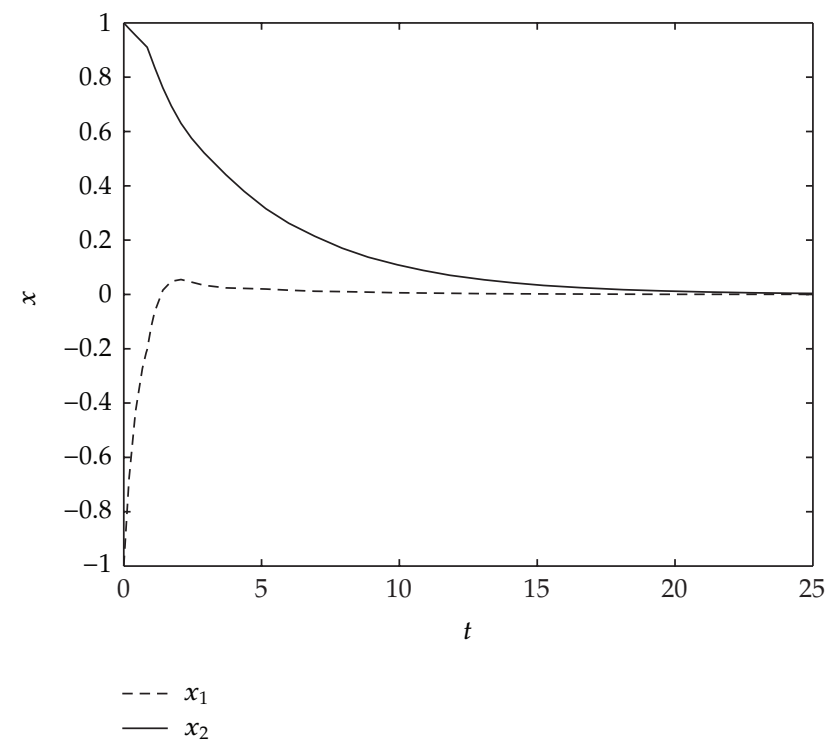

Figure 3: The trajectory of solution of system $i=1$ under the feedback controller (4.4).

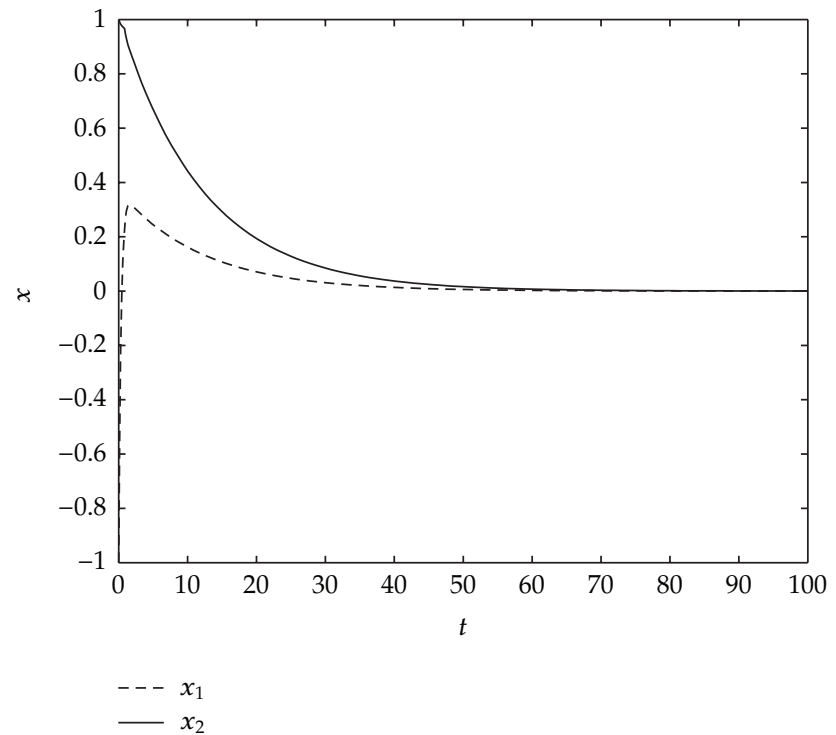

Figure 4: The trajectory of solution of system $i=2$ under the feedback controller (4.4). 
with

$$
\begin{array}{cc}
A_{1}=\left[\begin{array}{cc}
-2 & 1 \\
0 & 0.1
\end{array}\right], & D_{1}=\left[\begin{array}{cc}
0.1 & 0.1 \\
0.1 & -0.1
\end{array}\right], \quad B_{1}=\left[\begin{array}{c}
0 \\
0.1
\end{array}\right], \\
A_{2}=\left[\begin{array}{cc}
-3 & 0 \\
0 & 0.1
\end{array}\right], & D_{2}=\left[\begin{array}{cc}
-0.1 & 0.1 \\
0.1 & -0.1
\end{array}\right], \quad B_{2}=\left[\begin{array}{c}
0 \\
0.1
\end{array}\right], \\
E_{1, i}=E_{2, i}=E_{3, i}=\left[\begin{array}{cc}
0.02 & 0.01 \\
-0.01 & 0.01
\end{array}\right], & H_{1, i}=H_{2, i}=\left[\begin{array}{cc}
0.02 & 0 \\
0 & 0.01
\end{array}\right], \quad H_{3, i}=\left[\begin{array}{c}
0.01 \\
0
\end{array}\right], \quad i=1,2 .
\end{array}
$$

As an illustration, we choose $\alpha=0.9, \epsilon_{j, i}=1$ for $j=1,2, \ldots 6, i=1,2$,

$$
h(t)=0.1+|\sin t|, \quad h_{2}(t)=0.1+|\cos t|, \quad F_{1,1}(t)=F_{1,2}(t)=F_{2,1}(t)=F_{2,2}(t)=\left[\begin{array}{cc}
\sin t & 0 \\
0 & \sin t
\end{array}\right] .
$$

In this case, we can take $h_{1}=0.1, h_{M}=1.1$. Then, by using the LMI control toolbox in Matlab, solutions of LMIs (3.42) and (3.45) are given by

$$
\begin{aligned}
& P_{1}=\left[\begin{array}{ll}
0.0438 & 0.0046 \\
0.0046 & 0.0081
\end{array}\right], \quad Q_{1}=\left[\begin{array}{ll}
0.0153 & 0.0005 \\
0.0005 & 0.0001
\end{array}\right], \quad R_{1}=\left[\begin{array}{ll}
0.0037 & 0.0005 \\
0.0005 & 0.0010
\end{array}\right], \\
& U_{1}=\left[\begin{array}{cc}
0.0122 & -0.0005 \\
-0.0005 & 0.0006
\end{array}\right], \quad P_{2}=\left[\begin{array}{cc}
0.0272 & -0.0010 \\
-0.0010 & 0.0088
\end{array}\right], \quad Q_{2}=\left[\begin{array}{cc}
0.0059 & 0.0001 \\
0.0001 & 0.0001
\end{array}\right], \\
& R_{2}=\left[\begin{array}{cc}
0.0006 & -0.0002 \\
-0.0002 & 0.0022
\end{array}\right], \quad U_{2}=\left[\begin{array}{cc}
0.0038 & -0.0010 \\
-0.0010 & 0.0012
\end{array}\right] \text {, }
\end{aligned}
$$

with stabilizing controllers

$$
u_{1}(t)=[0.6907-6.5520] x(t), \quad u_{2}(t)=[-0.1997-5.6729] x(t)
$$

By computation, we obtain $\lambda^{*}=0.000007, \lambda_{1}=132.7976, \lambda_{2}=22.5048, \lambda_{3}=0.0444, h^{*}=0.5$. Thus, $\rho=7.8829 \times 10^{-5}$, and from (3.3) we obtain

$$
T_{0} \geq 2.3855 \times 10^{4}
$$

Hence, from Theorem 3.2, the switched systems (4.6) with arbitrary switching law subject to (4.12) are robustly stabilizable under the feedback controllers which are shown in (4.11).

By choosing the same initial condition as in Example 4.1, the trajectories of solutions of the switched system and the trajectories of solutions of subsystems 1 and 2 for this example are shown in Figures 5, 6, 7, and 8. 


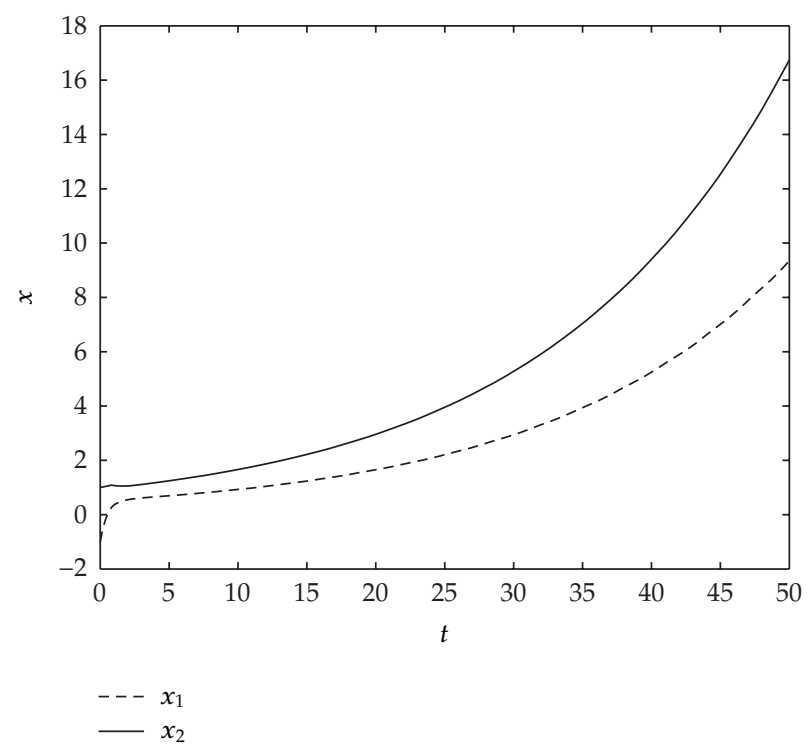

Figure 5: The trajectory of solution of system $i=1$.

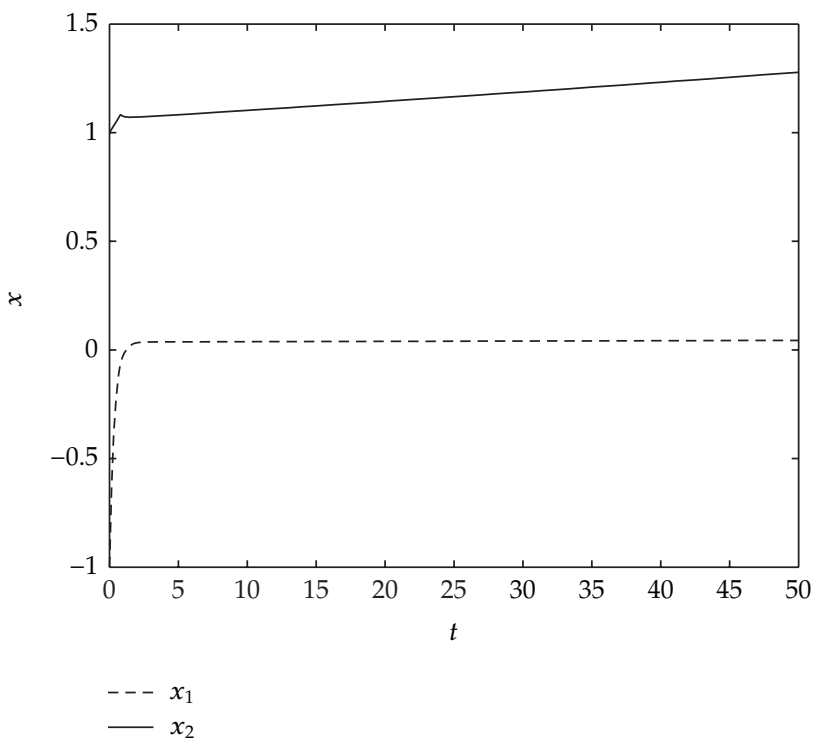

Figure 6: The trajectory of solution of system $i=2$.

\section{Conclusion}

In this paper, we study the problem of robust stabilization for a class of switched systems with time-varying delay. Comparing with some existing results in the literature, the novelty of our results is twofold. Firstly, the state delay is time-varying in which the restriction on the derivative of the time-delay function is not required to design switching rule for the robust 


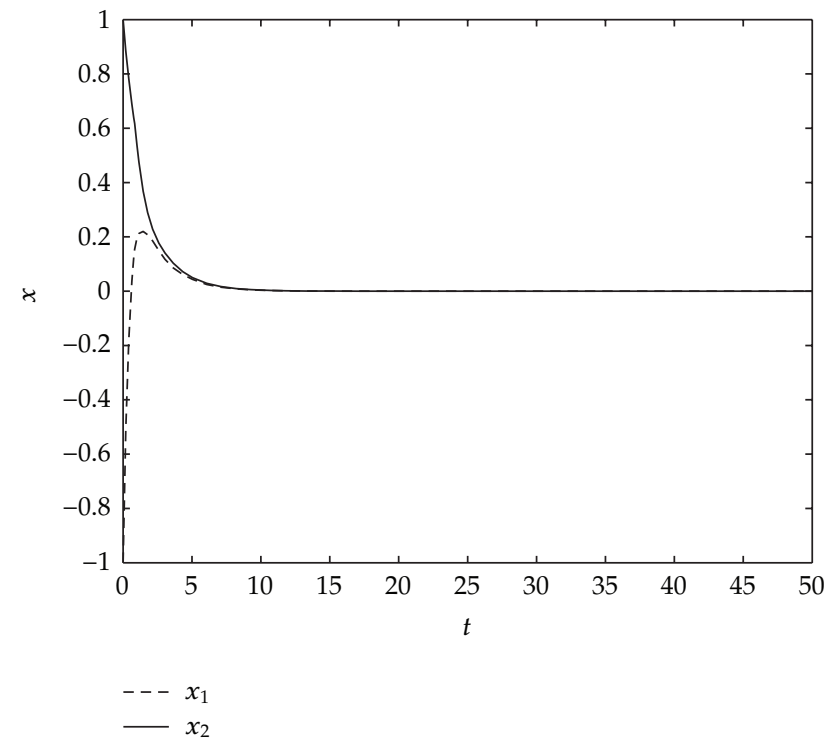

Figure 7: The trajectory of solution of system $i=1$ under the feedback controller (4.12).

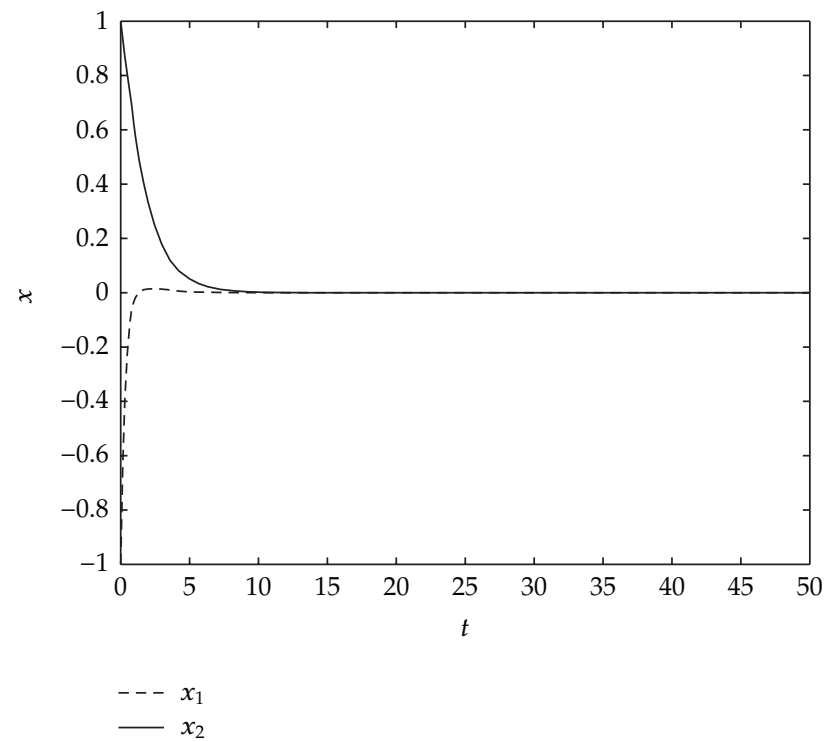

Figure 8: The trajectory of solution of system $i=2$ under the feedback controller (4.12).

stability of the system. Secondly, the obtained conditions for the robust stability are delaydependent and formulated in terms of the solution of standard LMIs which can be solved by various available algorithms. Numerical example is given to illustrate the effectiveness of the theoretical result. 


\section{Acknowledgments}

The first author is supported by the Graduate School, Chiang Mai University and Thai Government Scholarships in the area of science and technology (Ministry of Science and Technology). The second author is supported by the Center of Excellence in Mathematics, Thailand, and Commission for Higher Education, Thailand.

\section{References}

[1] P. J. Antsa, "A brief introduction to the theory and applications of hybrid systems," Proceedings of the IEEE, vol. 8, pp. 879-887, 2000.

[2] R. L. Bennett and G. E. Policello, "Switching systems in the 21st century," IEEE Communications Magazine, vol. 3, pp. 24-28, 1993.

[3] L. V. Hien, Q. P. Ha, and V. N. Phat, "Stability and stabilization of switched linear dynamic systems with time delay and uncertainties," Applied Mathematics and Computation, vol. 210, no. 1, pp. 223-231, 2009.

[4] L. V. Hien and V. N. Phat, "Exponential stabilization for a class of hybrid systems with mixed delays in state and control," Nonlinear Analysis. Hybrid Systems, vol. 3, no. 3, pp. 259-265, 2009.

[5] V. N. Phat, "Switched controller design for stabilization of nonlinear hybrid systems with timevarying delays in state and control," Journal of the Franklin Institute, vol. 347, no. 1, pp. 195-207, 2010.

[6] T. Botmart, P. Niamsup, and V. N. Phat, "Delay-dependent exponential stabilization for uncertain linear systems with interval non-differentiable time-varying delays," Applied Mathematics and Computation, vol. 217, no. 21, pp. 8236-8247, 2011.

[7] J. Liu, X. Liu, and W.-C. Xie, "Delay-dependent robust control for uncertain switched systems with time-delay," Nonlinear Analysis. Hybrid Systems, vol. 2, no. 1, pp. 81-95, 2008.

[8] P. Niamsup, "Stability of time-varying switched systems with time-varying delay," Nonlinear Analysis. Hybrid Systems, vol. 3, no. 4, pp. 631-639, 2009.

[9] V. N. Phat, T. Botmart, and P. Niamsup, "Switching design for exponential stability of a class of nonlinear hybrid time-delay systems," Nonlinear Analysis. Hybrid Systems, vol. 3, no. 1, pp. 1-10, 2009.

[10] Y. Zhang, X. Liu, and X. Shen, "Stability of switched systems with time delay," Nonlinear Analysis. Hybrid Systems, vol. 1, no. 1, pp. 44-58, 2007.

[11] Y. He, Q.-G. Wang, C. Lin, and M. Wu, "Delay-range-dependent stability for systems with timevarying delay," Automatica, vol. 43, no. 2, pp. 371-376, 2007.

[12] M. Wu, Y. He, J.-H. She, and G.-P. Liu, "Delay-dependent criteria for robust stability of time-varying delay systems," Automatica, vol. 40, no. 8, pp. 1435-1439, 2004.

[13] L. V. Hien and V. N. Phat, "New exponential estimate for robust stability of nonlinear neutral timedelay systems with convex polytopic uncertainties," Journal of Nonlinear and Convex Analysis, vol. 12 pp. 541-552, 2011.

[14] C.-H. Lien, K.-W. Yu, Y.-J. Chung, Y.-F. Lin, L.-Y. Chung, and J.-D. Chen, “Exponential stability analysis for uncertain switched neutral systems with interval-time-varying state delay," Nonlinear Analysis. Hybrid Systems, vol. 3, no. 3, pp. 334-342, 2009.

[15] M. S. Mahmoud and F. M. AL-Sunni, "Interconnected continuous-time switched systems: robust stability and stabilization," Nonlinear Analysis. Hybrid Systems, vol. 4, no. 3, pp. 531-542, 2010.

[16] Z. Zhang and X. Liu, "Robust stability of uncertain discrete impulsive switching systems," Computers E Mathematics with Applications, vol. 58, no. 2, pp. 380-389, 2009.

[17] G. Zong, S. Xu, and Y. Wu, "Robust $H_{\infty}$ stabilization for uncertain switched impulsive control systems with state delay: an LMI approach," Nonlinear Analysis. Hybrid Systems, vol. 2, no. 4, pp. 1287-1300, 2008.

[18] S. Boyd, L. E. Ghaoui, E. Feron, and V. Balakrishnan, Linear Matrix Inequalities in System, SIAM, Philadelphia, Pa, USA, 1994.

[19] X.-M. Sun, J. Zhao, and D. J. Hill, "Stability and $L_{2}$-gain analysis for switched delay systems: a delaydependent method," Automatica, vol. 42, no. 10, pp. 1769-1774, 2006.

[20] W. Zhang, X.-S. Cai, and Z.-Z. Han, "Robust stability criteria for systems with interval time-varying delay and nonlinear perturbations," Journal of Computational and Applied Mathematics, vol. 234, no. 1, pp. 174-180, 2010. 
[21] D. Wang and W. Wang, "Delay-dependent robust exponential stabilization for uncertain systems with interval time-varying delays," Journal of Control Theory and Applications, vol. 7, no. 3, pp. 257-263, 2009.

[22] Y. G. Sun, L. Wang, and G. Xie, "Stability of switched systems with time-varying delays: Delaydependent common Lyapunov functional approach," in Proceedings of the American Control Conference, vol. 5, pp. 1544-1549, 2006. 


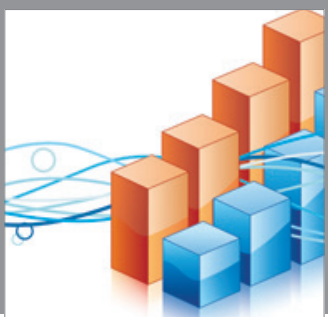

Advances in

Operations Research

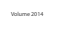

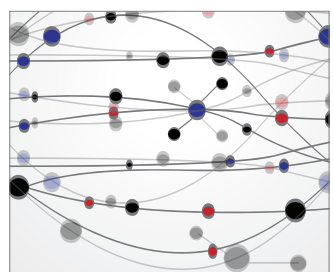

\section{The Scientific} World Journal
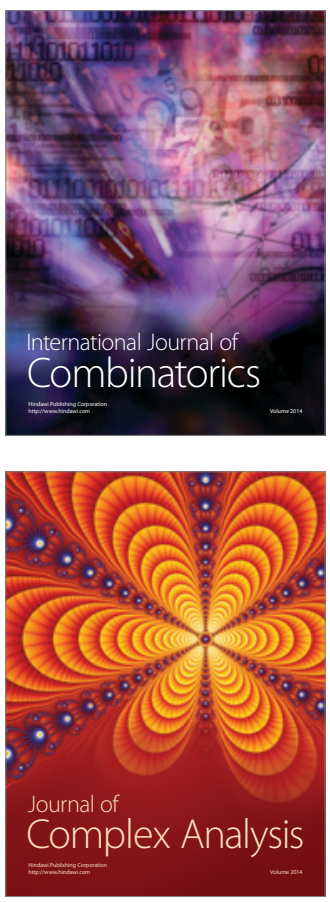

International Journal of

Mathematics and

Mathematical

Sciences
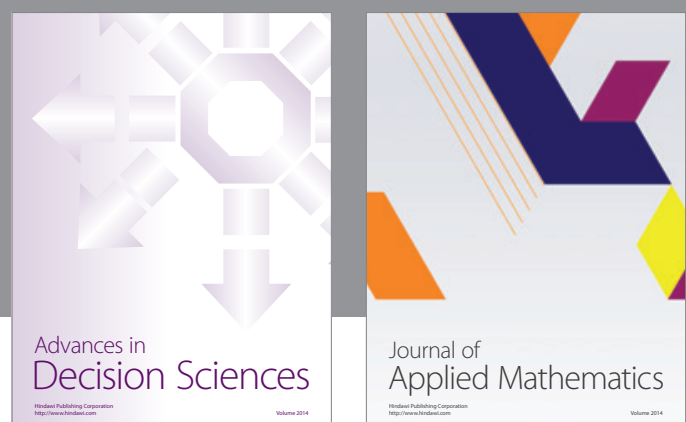

Journal of

Applied Mathematics
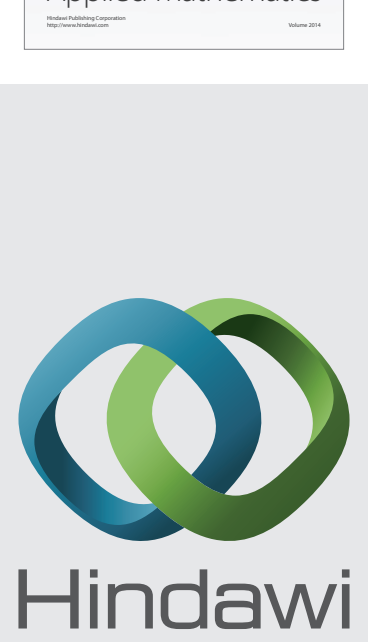

Submit your manuscripts at http://www.hindawi.com
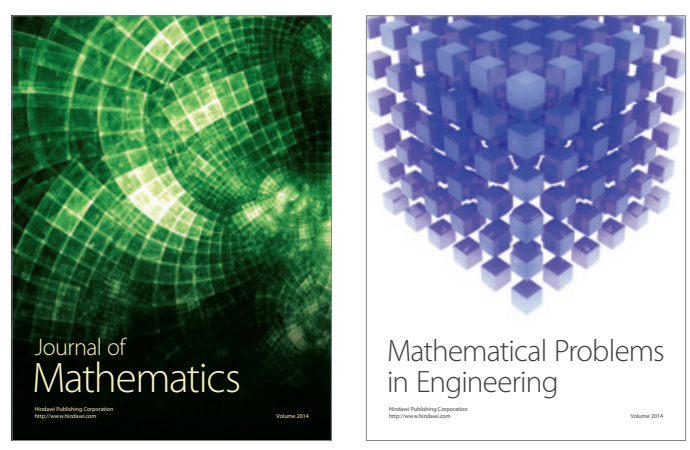

Mathematical Problems in Engineering
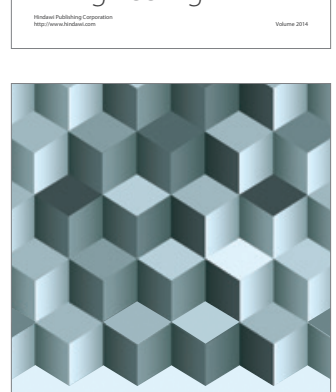

Journal of

Function Spaces
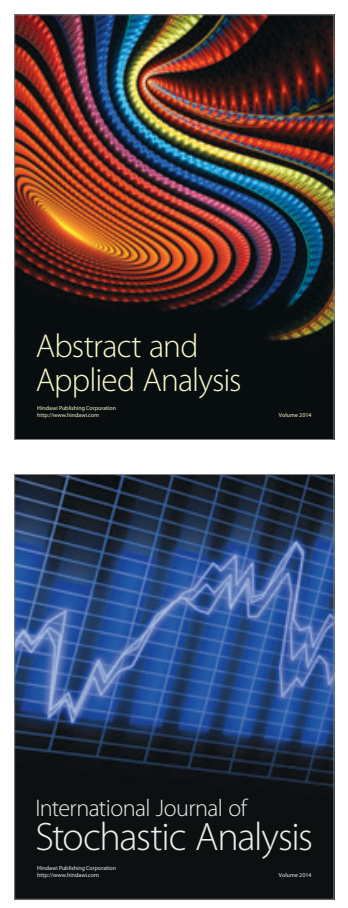

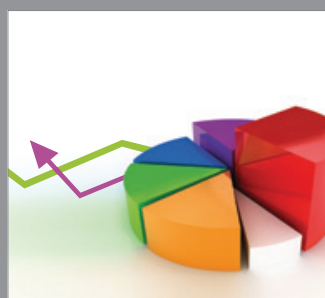

ournal of

Probability and Statistics

Promensencen
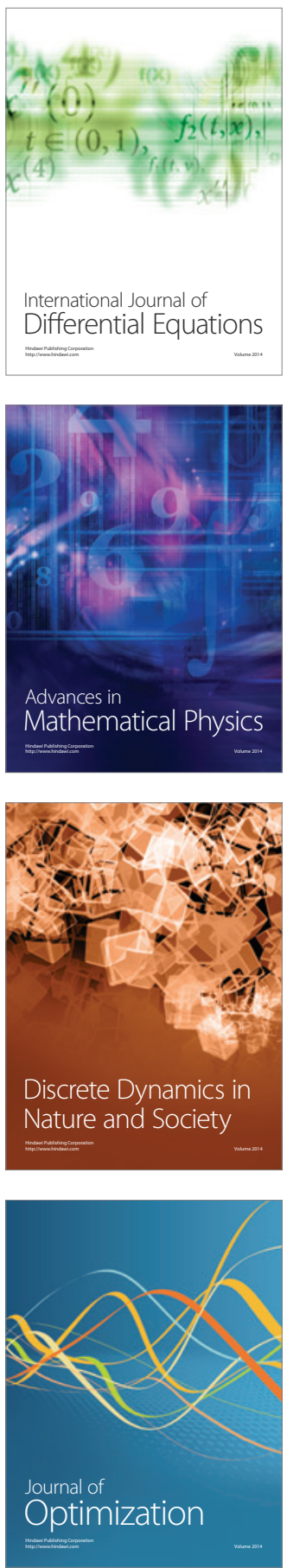